\title{
Research Paper \\ Effect of 6 Weeks of Aerobic Training on TGF-B1, Myostatin and Matrix Metallo- proteinase 9 Genes Expression in the Tendon of Fast- and Slow-Twitch Muscles of Male Wistar Rats
}

\author{
Ghasem Mohammadnezhad ${ }^{1} \odot$, *Hasan Matin Homaee ${ }^{1} \odot$,Farshad Ghazalian ${ }^{2} \odot$
}

1. Department of Exercise Physiology, Central Tehran Branch, Islamic Azad University, Tehran, Iran.

2. Department of Exercise Physiology, Science and Research Branch, Islamic Azad University, Tehran, Iran.

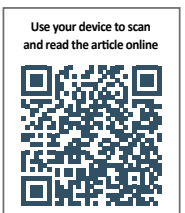

Cittation: Mohammadnezhad Gh, Matin Homaee H, Ghazalian F. [Effect of 6 Weeks of Aerobic Training on TGF-B1, Myostatin and Matrix Metalloproteinase 9 Genes Expression in the Tendon of Fast- and Slow-Twitch Muscles of Male Wistar Rats (Persian)]. Journal of Arak University of Medical Sciences (JAMS). 2020; 23(3):278-291. https://doi.org/10.32598/JAMS.23.3.5849.2

Key words: Aerobic training, Tendon, TGF- $\beta 1$, MMP9,

Myostatin

\section{ABSTRACT}

Article Info:

Received: 15 Mar 2020

Accepted: 17 May 2020

Available Online: 01 Aug 2020
Background and Aim Tendon, like the skeletal muscle, exhibits mechanical and morphological adaptations resulted from exercise training; however, little is known about the basic cellular and molecular mechanisms that regulate these responses. The aim of the present study was therefore to investigate the effect of 6 weeks of aerobic training on the TGF- $\beta 1$, myostatin and MMP9 mRNAs expression in the tendon of fast- and slow-twitch muscles.

Methods \& Materials For this purpose, 12 male Wistar rats at 8 weeks of age were randomly divided into two groups: experimental $(n=6)$ and control $(n=6)$. The exercise group performed aerobic training for 6 weeks, 5 sessions per week. Forty-eight hours after the last training session, all rats were sacrificed and the tendons of soleus and Extensor Digitorum Longus (EDL) muscles were extracted. Expression of TGF- $\beta 1$, myostatin and MMP9 mRNAs were assayed using RealTime-PCR. Independent t-test was also used for statistical analysis.

Ethical Considerations All stages of the study were conducted according to the ethical guidelines and authorization of Research Deputy of Islamic Azad University, Central Tehran Branch No. IR.IAU.PS.REC.1398.296. Results The results showed that the expression of TGF- $\beta 1$ mRNA in EDL and soleus tendons significantly increased $(P \leq 0.001)$, whereas the expression of myostatin in EDL tendon was significantly reduced ( $P \leq$ 0.001). Increased mRNA expression of MMP9 in the tendon of EDL and soleus muscles was not statistically significant $(\mathrm{P}>0.05)$.

Conclusion It seems that aerobic exercise can modulate the expression of genes involved in the regulation of tendon collagen in a muscle type-dependent manner.

\section{Extended Abstract}

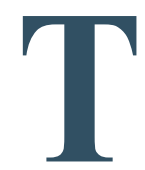

\section{Introduction}

endon tissue along the Extracellular Matrix

(ECM) is a muscle that mechanically and structurally adapts to the muscle with mechanical load [2]. Although the mechanical and morphological changes that occur in tendons in response to resistance exercise are well documented, little is known about the underlying cellular and molecular mechanisms that regulate these responses.

-

* Corresponding Author:

Hasan Matin Homaee, PhD.

Address: Department of Exercise Physiology, Central Tehran Branch, Islamic Azad University, Tehran, Iran. Tel: +98 (912) 3680810

E-mail: hasanmatinhomaee@gmail.com 
The "Transforming Growth Factor Beta 1 (TGF- $\beta 1$ ) family" messaging pathway appears to play a major role in tendon adaptation to resistance exercise. Myostatin is a member of the large TGF- $\beta$ family, the expression of which negatively regulates skeletal muscle growth [5].

Both TGF- $\beta 1$ and myostatin stimulate tendon fibroblast proliferation and type I collagen synthesis [4, 6]. Matrix Metalloproteinases (MMPs) are zinc-dependent endopeptidases that break down collagen and other structural molecules. MMPs are essential for baseline ECM homeostasis. The expression of MMPs is regulated by various cytokines and messenger molecules, including TGF- $\beta 1$ [2].

However, the exact components of the signal transduction pathways that regulate MMP expression following exercise have not yet been fully elucidated. Therefore, aim of the present study was to investigate the effect of 6 weeks of aerobic exercise on the expression of TGF- $\beta 1$, myostatin and MMP9 genes in the fast and slow-twitch muscle tendons of male Wistar rats.

\section{Materials and Methods}

Twelve male Wistar rats (8-week-old) were randomly divided into 2 groups of "exercise" $(n=6)$ and "control" $(n=6)$. The exercise group performed aerobic exercise for 6 weeks, which included 5 sessions of treadmill running per week for 6 weeks [13].

Forty-eight hours after the last training session, all rats were killed. Then, the Soleus Tendon (SOL) and the Extensor Digitorum Longus muscle (EDL) of their right foot were immediately and carefully extracted and kept at minus $80^{\circ} \mathrm{C}$ for further measurements. The mRNA expression

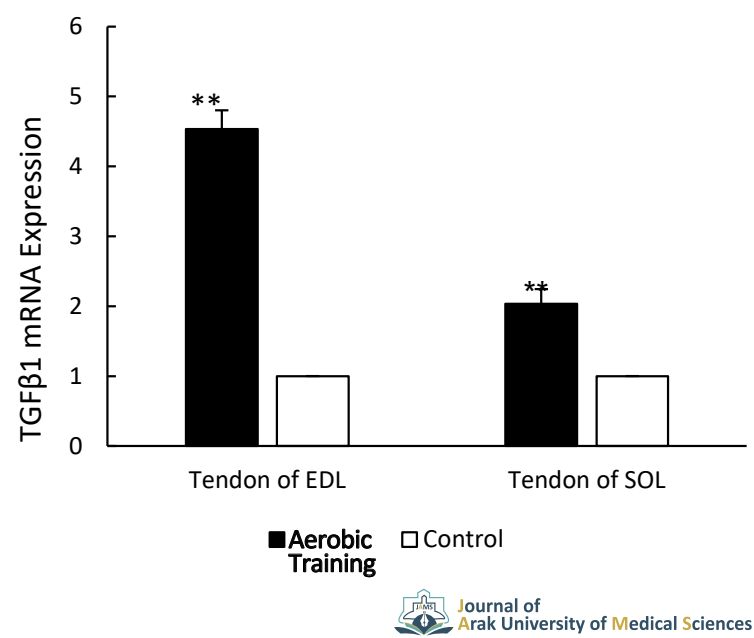

Figure 1. TGF- $\beta 1$ mRNA expression in EDL and soleus muscles $* * * \mathrm{P} \geq 0.001$ levels of TGF- $\beta 1$, myostatin and MMP9 genes were measured using Real time-PCR. Independent t-test was used to analyze the obtained data.

\section{Results}

The results showed that there was a significant difference between the TGF- $\beta 1$ gene values of EDL and soleus muscles in the aerobic exercise group compared to the control group. TGF- $\beta 1$ gene expression in EDL and soleus muscles was significantly increased in the aerobic exercise group compared to the control group (EDL muscle: $0.63 \pm 0.09$ compared to $0.14 \pm 0.04, \mathrm{P} \geq 0.001$, and soleus muscle: $0.36 \pm 0.08$ compared to $0.17 \pm 0.04, \mathrm{P} \geq 0.001$ ) (Figure 1).

The results of independent t-test showed that myostatin gene expression was significantly reduced only in the EDL muscle of the aerobic exercise group compared to the control group (EDL muscle: $0.3 \pm 0.1$ compared to $0.58 \pm 0.07$, $\mathrm{P} \geq 0.001$, and soleus muscle: $0.20 \pm 0.09$ compared to $0.29 \pm 0.05, \mathrm{P} \geq 0.001$ ) (Figure 2).

Also, no significant difference was observed between MMP9 gene expression in EDL and soleus muscles between the aerobic exercise and control groups (EDL muscle: $0.21 \pm 0.08$ compared t o $0.15 \pm 0.02, \mathrm{P}>0.05$, and soleus muscle: $0.27 \pm 0.07$ compared to $0.19 \pm 0.1$, $\mathrm{P}>0.05$ ) (Figure 3).

\section{Discussion}

The results of the present study showed that following a 6-week aerobic exercise program, TGF- $\beta 1$ mRNA expres-

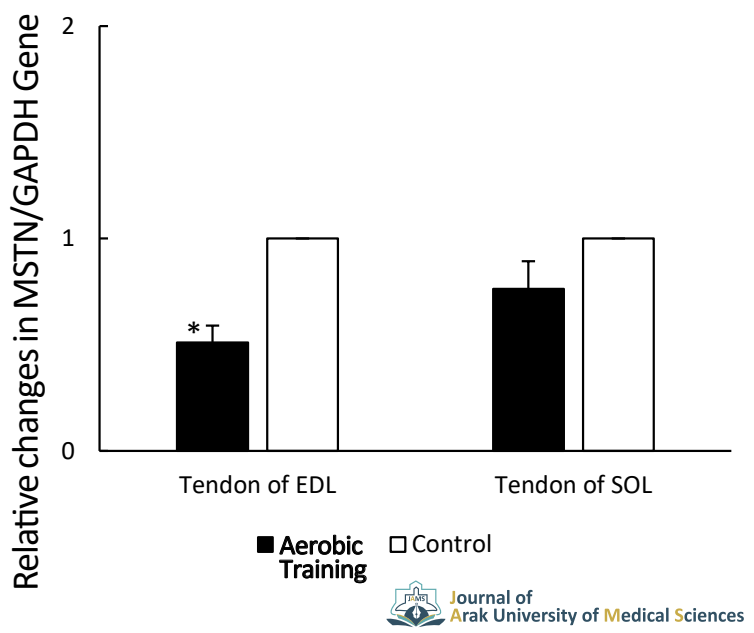

Figure 2. Myostatin mRNA gene expression in EDL and soleus muscles

$* * * \mathrm{P} \geq 0.001$ 


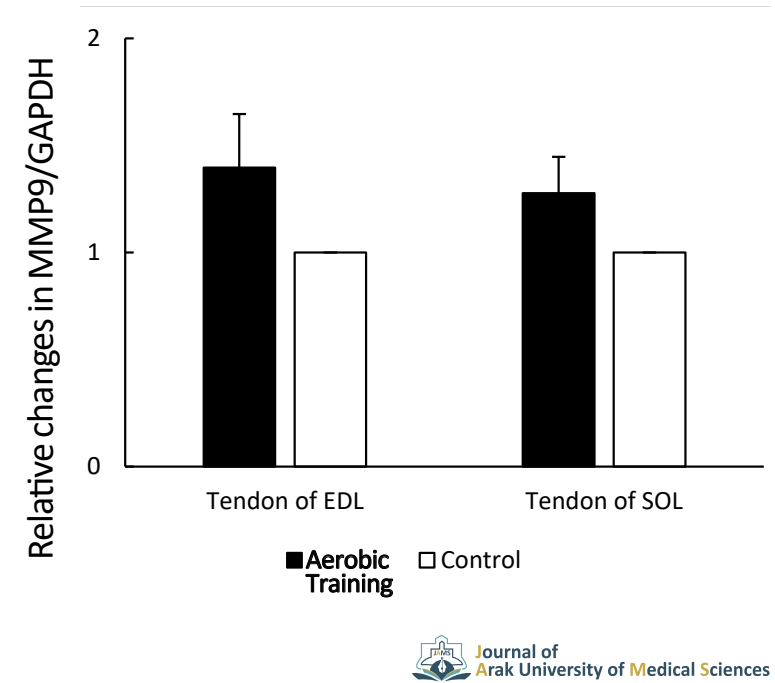

Figure 3. MMP9 mRNA gene expression in EDL and soleus muscles

sion increased in both EDL and soleus muscles. Heniemir et al. (2007) reported increased mRNA levels of TGF- $\beta 1$ genes and type I and III collagens in the gastrocnemius muscle and Achilles tendon following isometric, concentric and eccentric contractions by stimulating the sciatic nerve for 4 days [15].

Also, another study showed that long-term endurance training caused a significant increase in TGF- $\beta 1 \mathrm{mRNA}$ in soleus muscle [8]. The study showed that the antioxidant status of muscle affected the expression of TGF- $\beta 1$ [16]. Evidence suggests that TGF- $\beta 1$ is the main mediator of inducing collagen synthesis in fibroblasts through mechanical loading [15] and a similar role for TGF- $\beta 1$ in tendons has been suggested [17].

An increase of more than $453 \%$ in the expression of TGF- $\beta 1$ mRNA in the EDL muscle tendon, compared with a $203 \%$ increase in its expression in the soleus tendon following aerobic exercise - observed in the present study is more likely to indicate the involvement of fast-twitch muscles in aerobic exercise, which are less used in normal daily practices.

There is much evidence to support the idea that myostatin regulation is specific to the type of muscle fibers and is strongly associated with myosin heavy chain IIb isoform [21], and High concentrations of myostatin protein have been observed in fast-twitch muscle compared to slowtwitch fibers [22].

These reports could justify the results of the present study, which showed a more than $49 \%$ reduction in myostatin mRNA expression in the EDL muscle compared with a slight $24 \%$ reduction in the expression of this gene in the soleus muscle following aerobic exercise.

Exercise appears to damage collagen tissue in skeletal muscle and tendons, and MMP9 levels increase to remove these damaged elements from tissue. In addition, MMPs have been reported to be essential for the induction of exercise-induced angiogenesis in skeletal muscle [25].

In the present study, exercise increased MMP9 expression by $40 \%$ in EDL muscles and by $28 \%$ in soleus muscles; but these changes were not statistically significant. Consistent with the results of the present study, Rollman et al. (2009) reported a non-significant increase in MMP9 expression in the human vastus lateralis muscle following aerobic exercise [26].

The decrease in myostanin levels following aerobic exercise observed in the present study can be considered as a mechanism for the relative increase in MMP9 mRNA expression in tendon tissue. Mendias et al. (2015) reported a $141 \%$ increase in MMP9 gene expression in the tendon tissue of myostatin-free rats (rats whose myostatin was genetically inactivated) [18].

In addition, activation of the ERK1/2 and JNK-NF- $\mathrm{BB}$ signaling pathways via ROS has been shown to be essential for the positive regulation/activation of MMP9 and cell migration induced by TGF- $\beta 1$ [27], which can be another mechanism to justify the relative increase in MMP9 mRNA expression in tendon tissue in this study.

\section{Conclusion}

In general, aerobic exercise positively regulates basal levels of TGF- $\beta 1$ gene and negatively regulates basal levels of myostatin gene in fast- and slow-twitch muscle tendons; and these effects are significantly greater in fast-twitch muscle tendons. It seems that aerobic exercise can modulate the expression of genes Involved in the regulation of tendon tissue collagen in a muscle-dependent manner.

\section{Ethical Considerations}

\section{Compliance with ethical guidelines}

All stages related to animals were performed in accordance with Ethical Instructions and after obtaining license (Code: IR.IAU.PS.REC.1398.296) from the Vice Chancellor for Research, Islamic Azad University Central Tehran Branch. 
Funding

The present paper was extracted from the $\mathrm{PhD}$. thesis of the second author, Department of Sports Physiology, Faculty of Physical Education and Sports Sciences, Islamic Azad University, Central Tehran Branch.

\section{Authors' contributions}

Conceptualization, review, subject definition, writing review \& editing: All authors; Methodology, data analysis: Ghasem Mohammadnejad.

\section{Conflicts of interest}

The authors declared no conflict of interest.

\section{Acknowledgements}

The authors would like to thank Dr. Reza Gharakhanloo, Department of Physical Education and Sports Science, Faculty of Human Sciences, Tarbiat Modares University, and Faculty of Human Sciences for their cooperation in conducting this study. 
This Page Intentionally Left Blank 


\title{
اثر 7 هفته تمرين هوازى بر بيان رثنهاى TGF-B1، ميوستاتين و ماتريكس متالويروتئيناز 9 در تاندون عضلات تند و كندانقباض موش هوش بران نر ويستار
}

\author{
قاسم محمدنزاد' ه ، "حسن متينهمائى' ـ، فرشاد غزاليان'
}

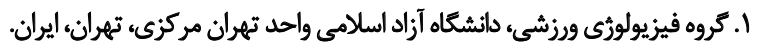

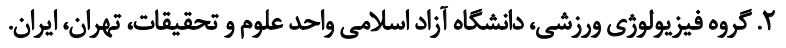

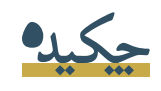

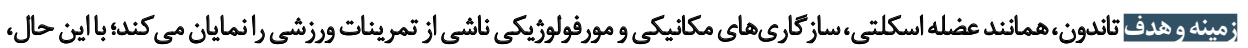

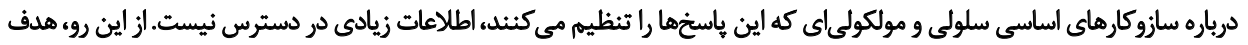

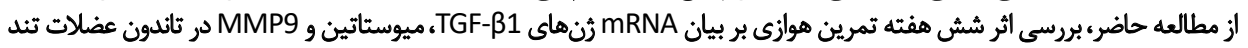
وكندانقباض بود.

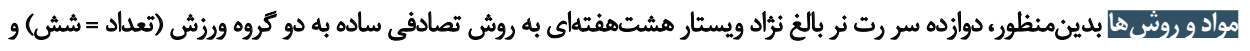

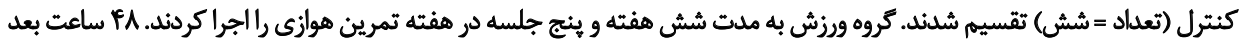

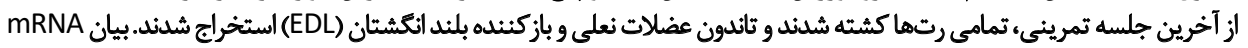

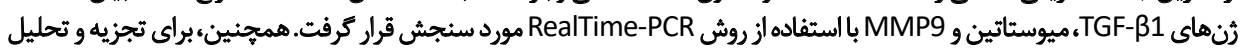
آمارى، ازٔز آزمون تى مسثقل استفاده شد.

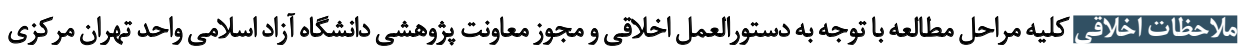

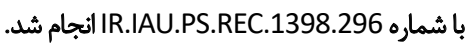

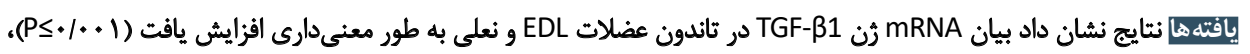

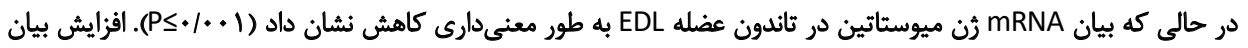

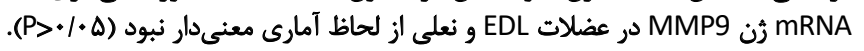

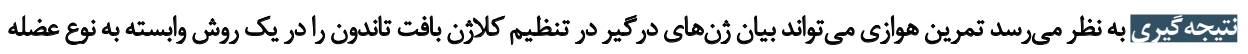
تعديل كند.
\end{abstract}

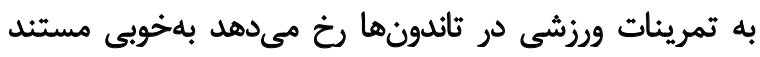

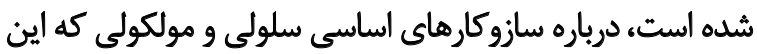

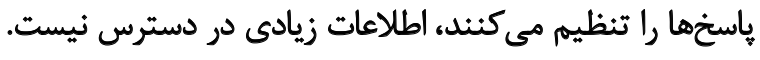

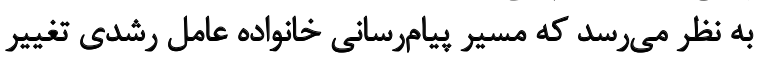

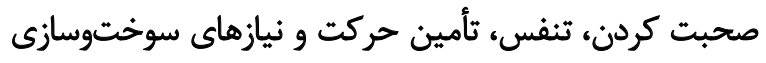

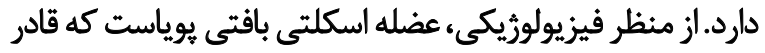

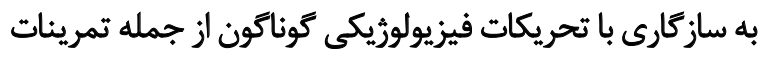

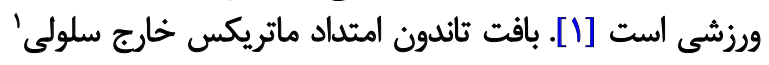

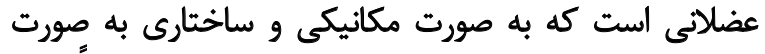

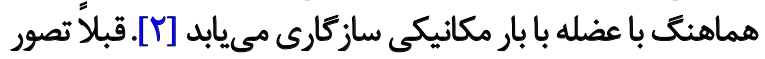

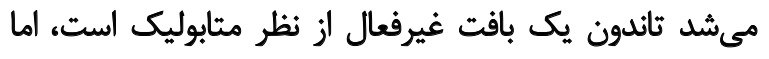

1. Extracellular Matrix (ECM) 


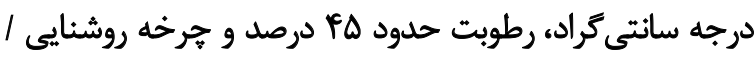

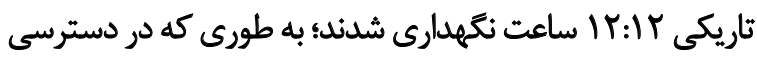

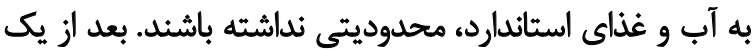

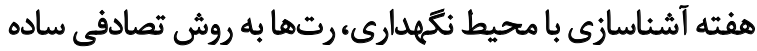
به دو كروه تقسيم شدند: كروه وريط ورز

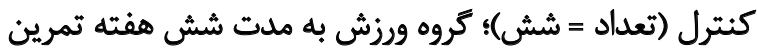

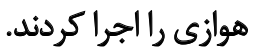

قبل از اجراى يروتكل تمرينى، تمامى حيوانات به مدت يك بردي

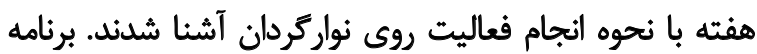

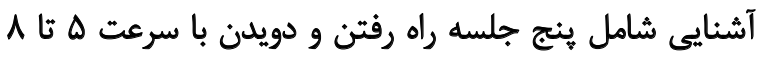

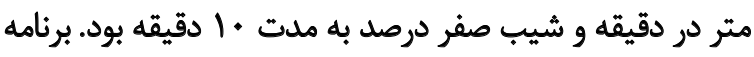

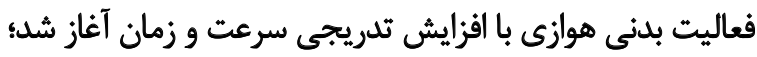

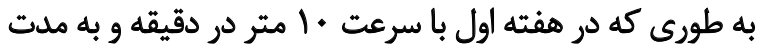

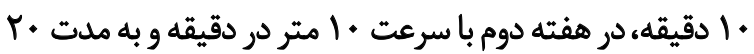

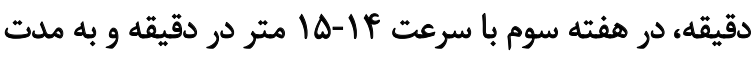

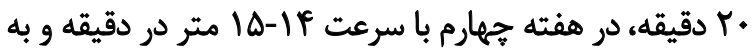

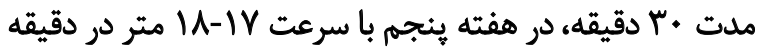

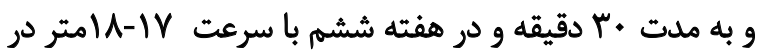
دقيقه و به مدت ·f د دقيقه بود.

در ابتدا و انتهاى هر جلسه تمرينى ب دقيقه گرم كردن و و

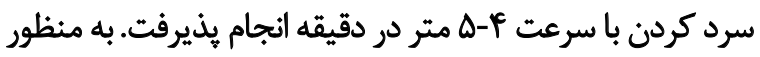

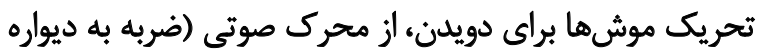

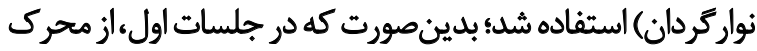

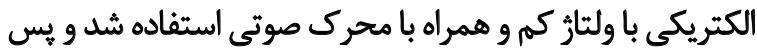

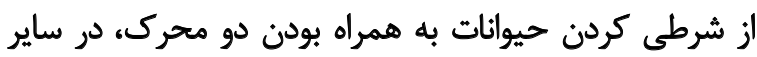

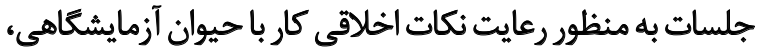
فقط از محرك صوتى استفاده شد [III)].

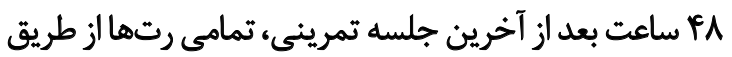

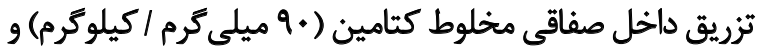

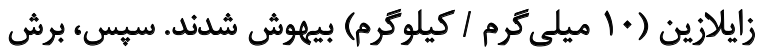

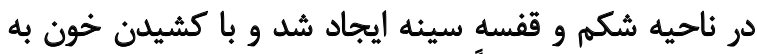

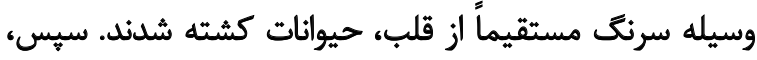

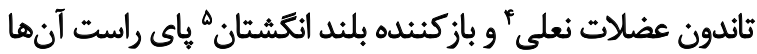

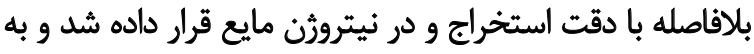

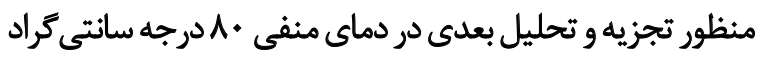

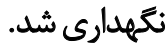

به منظور استخراج RNA تام از بافت تاندون هموزنشده، 1

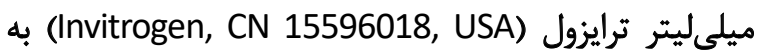

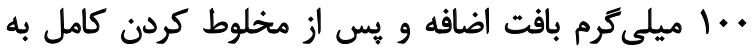

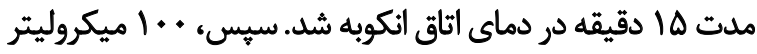

4. Soleus

5. Extensor digitorum longus (EDL)
شكل دهنده بتا | (TGF-B1) نقش اصلى رادر سازكارى تاندون

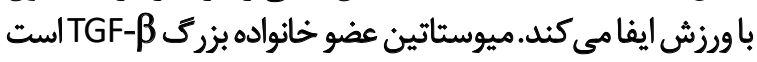

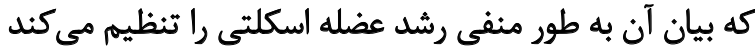

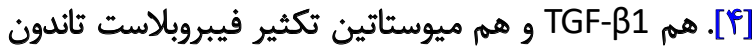

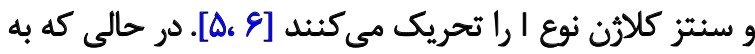

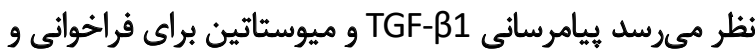

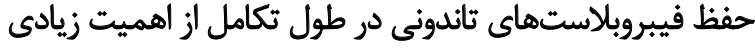

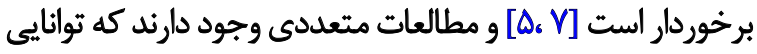

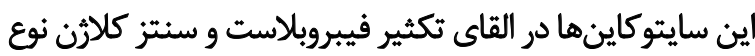

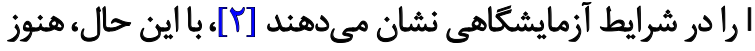

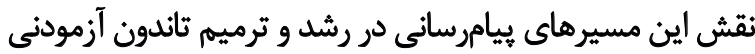

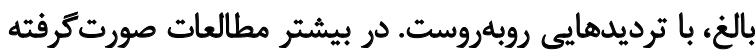

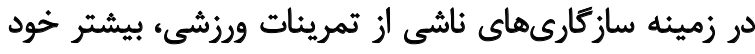

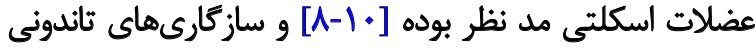

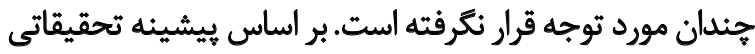

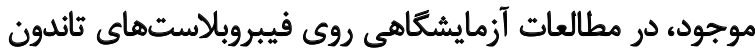

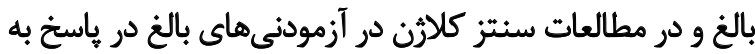

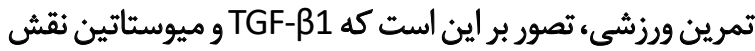

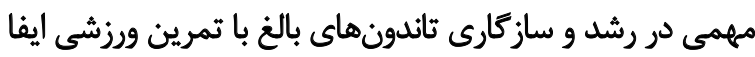

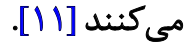

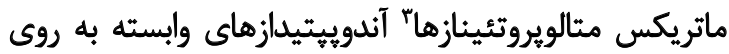

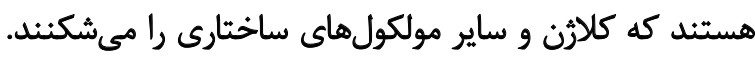

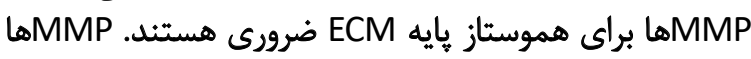

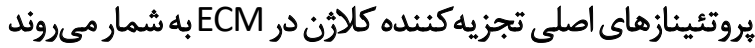

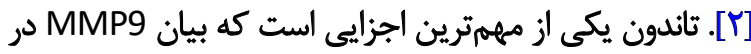

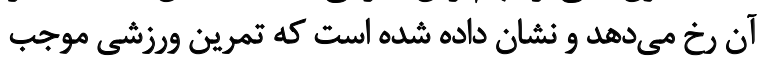
تحريك سلولهاى تاندون براى بيان MMP9 مئش

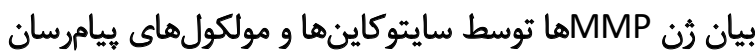

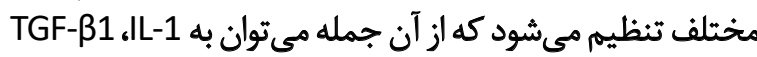
TNF- $\alpha$

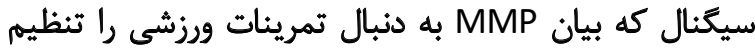

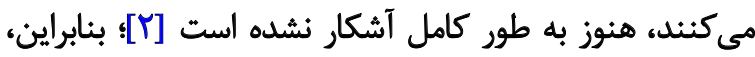

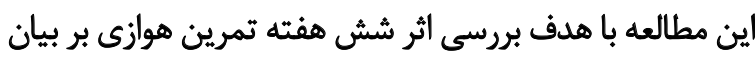

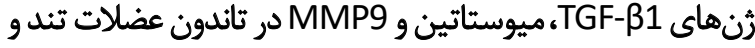
كندانقباض موشهاي صحرايى نر نرّاد ويستار انجام شد.

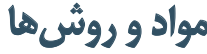

يُروهش حاضر از نوع تجربى است كه به شيوه آزمايشكاهي

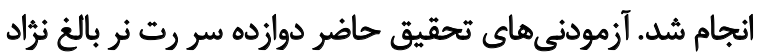

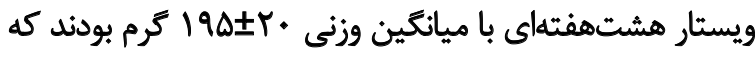

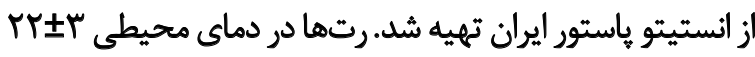

2. Transforming growth factor- $\beta 1$

3. Matrix metalloproteinases (MMPs) 
جدول ا. توالى يرايمرهاى موردمطالعه در تحقيق حاضر

\begin{tabular}{|c|c|c|c|c|c|}
\hline Accession Number & $\begin{array}{l}\text { Product } \\
\text { Length }\end{array}$ & Tm & Primer (5' 3') & Forward/Reverse & Gene \\
\hline \multirow{2}{*}{ NM_021578.2 } & \multirow{2}{*}{ r.. } & $\Delta Q / I V$ & CAACAACGCAATCTATGACAA & $F$ & \multirow{2}{*}{ TGF- $\beta 1$} \\
\hline & & $\Delta F / \Delta V$ & CAAGGTAACGCCAGGAAT & $\mathrm{R}$ & \\
\hline \multirow{2}{*}{ NM_019151.1 } & \multirow{2}{*}{$\mathrm{vi}$} & $\Delta r / \& \Delta$ & CTACCACGGAAACAATCATTA & $\mathbf{F}$ & \multirow{2}{*}{ Myostatin } \\
\hline & & $\Delta q / r$ & AGCAACATTTGGGCTTTCCAT & $\mathbf{R}$ & \\
\hline \multirow{2}{*}{ NM_031055.1 } & \multirow{2}{*}{109} & $\Delta \mathcal{N} Q \mathcal{C}$ & CCACCGAGCTATCCACTCAT & $\mathbf{F}$ & \multirow{2}{*}{ MMP-9 } \\
\hline & & $\Delta V / M I$ & GTCCGGTITCAGCATGTITI & $\mathbf{R}$ & \\
\hline \multirow{2}{*}{ XM_017593963.1 } & \multirow{2}{*}{$|r|$} & \&I/AR & AAGTTCAACGGCACAGTCAAGG & $F$ & \multirow{2}{*}{ GAPDH } \\
\hline & & EI/M & CATACTCAGCACCAGCATCACC & $\mathbf{R}$ & \\
\hline
\end{tabular}

هرحله سوم، به مدت ه ثانيه در دماي A درجه سانتي

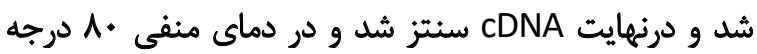

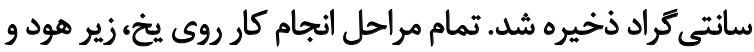

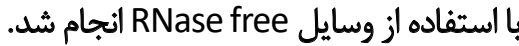

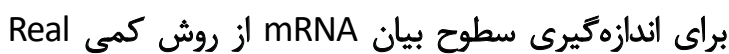

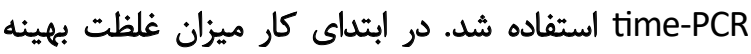

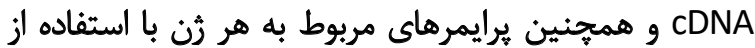

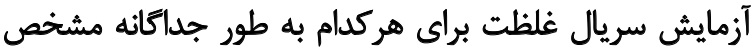

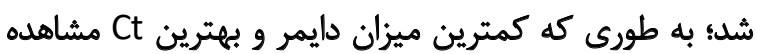

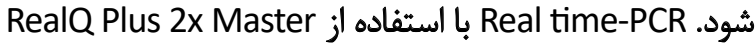
AMPLIQON شركت Mix Green نانوكرم از cDNA انجام كرفت.

توالى ثرايمرهاى مربوط به متغيرهاى موردمطالعه بر اساس

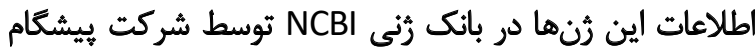

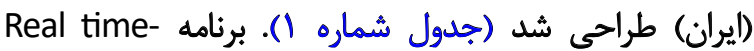

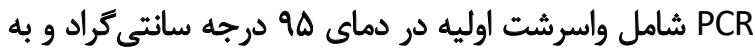

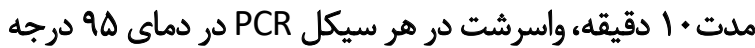

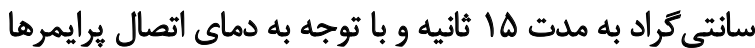

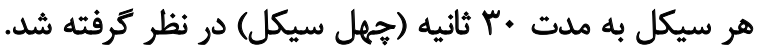

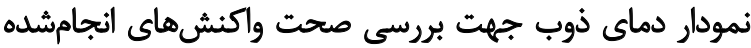

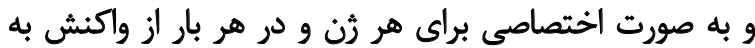

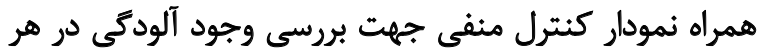

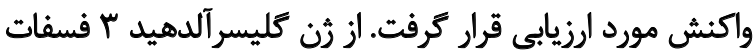

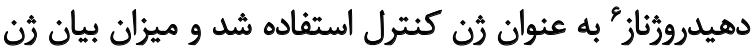

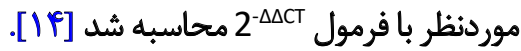
براى بررسى طبيعى بودن توزيع دادهها از آزمون كولموكروف

6. Glyceraldehyde-3-phosphate dehydrogenase (GAPDH)
كلروفرم سرد (Merek, CAS 67-66-3 102445, Germany)

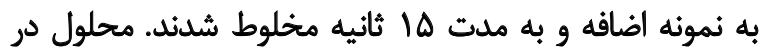

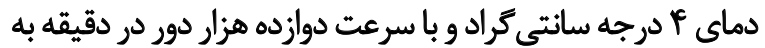

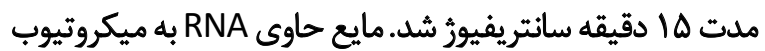

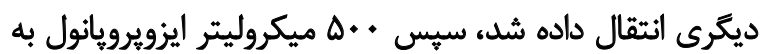

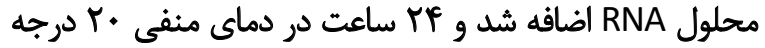

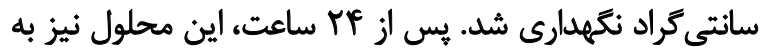

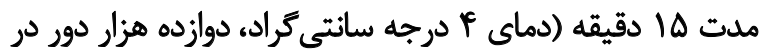

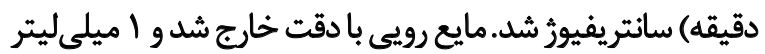

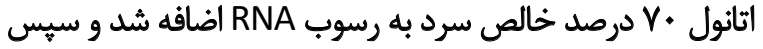

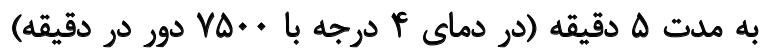

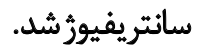

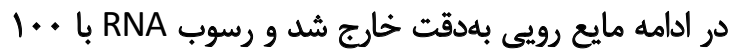

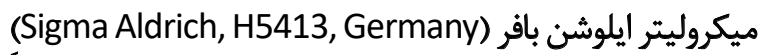

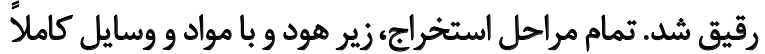

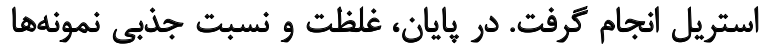
با استفاده از دستكاه اسيكتوفتومتر (Eppendorf, Germany)

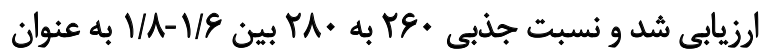
تخليص مطلوب تعريف شد.

يس از استخراج RNA با خلوص و غلظت بالا، مراحل سنتز

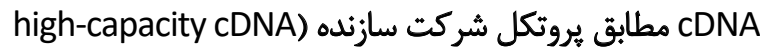
آب بام (reverse transcription kit

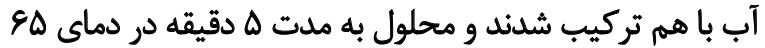

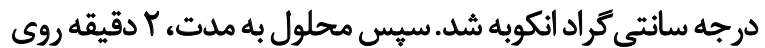

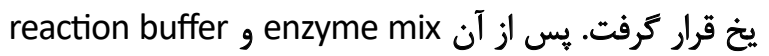

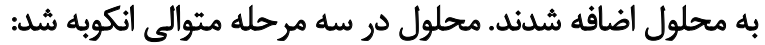

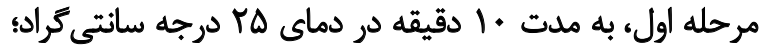

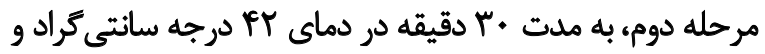


حاضر نشان داد به دنبال شش هفته برنامه تمرين هوازى دويدن

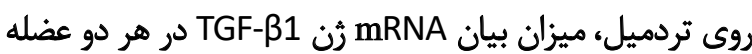

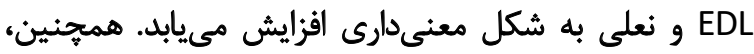

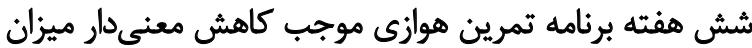

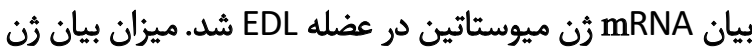

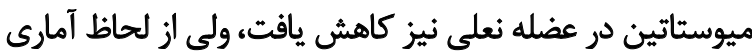

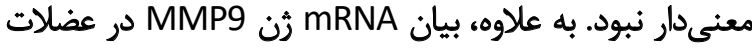
EDL و نعلى افزايش غيرمعنى دار نشان داد.

مرور متون يُروهشى حاكى از آن است كه بيشتر مطالعات

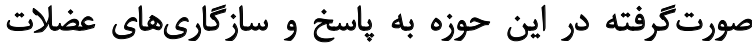

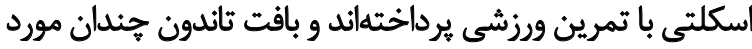

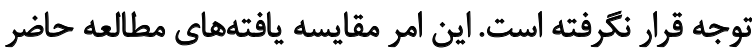

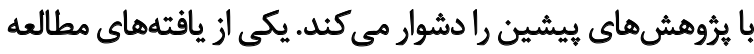

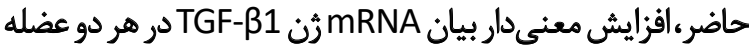

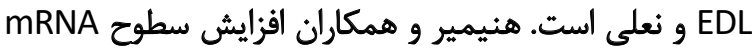

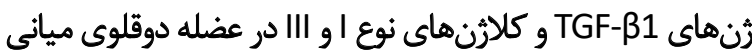

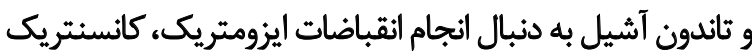

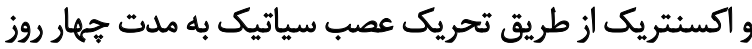

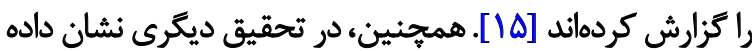

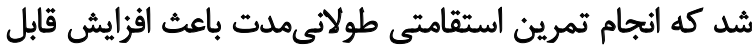

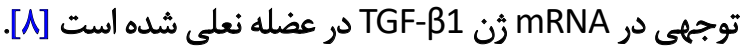
سازوكارهايى كه در طول تمرينات ورزشى بار مكانيكى را به

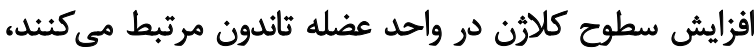

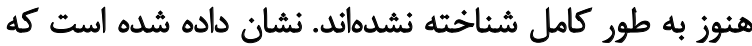

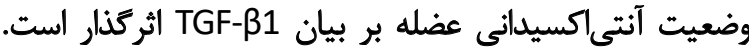

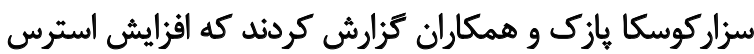
اكسيداتيو از طريق افزايش بيان NADPH اكسيداز و كاني كاهي

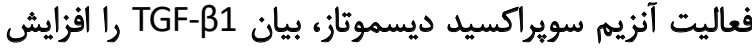
مى فهد [919].درحقيقت، TGF-B1 سايتوكاينى است كه خاصيت
اسميرنف استفاده شد. به منظور تحليل دادهها از آزمون تى

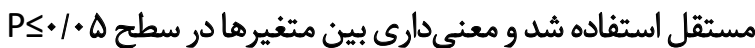

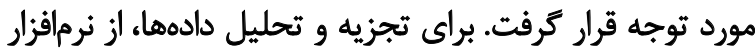

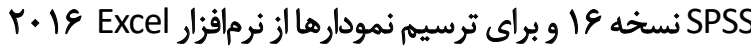

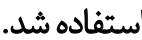

يافتهها

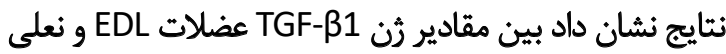

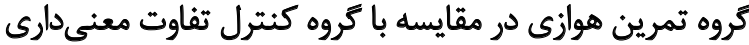

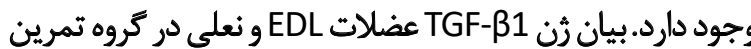

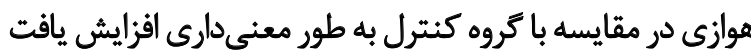

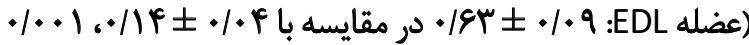

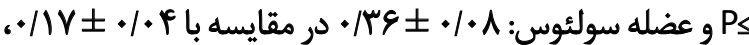

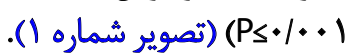

نتايج آزمون تى مستقل نشان داد بيان ثن ميوستاثين تنها در

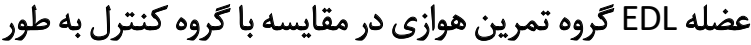

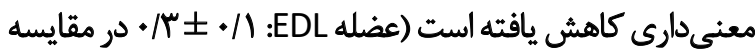

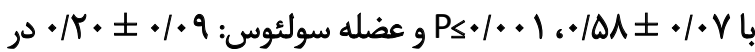

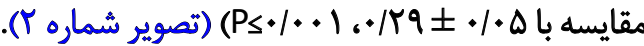
نتايج آزمون تى مستقل نشان داد تفاوت معنى دارى بين بيان ون

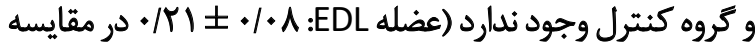

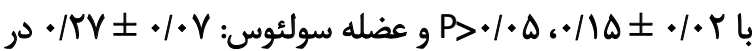

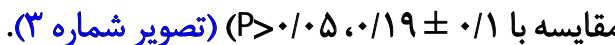
بحث مطالعه حاضر با هدف بررسى اثر شش هفته تمرين هوازى بر

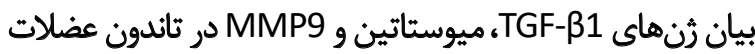
تند و كندانقباض موشهاى نر ويستار انجام شد. نتايج مطالعه

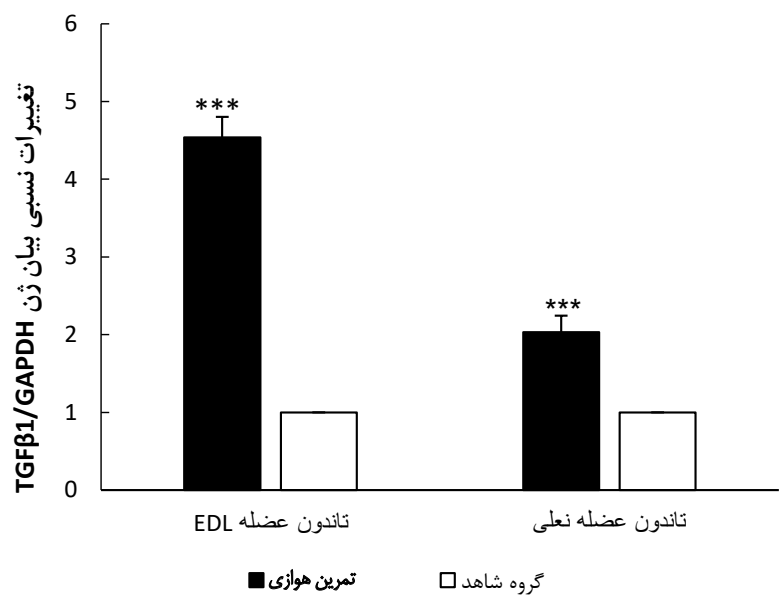

تصوير ا. ميزان بيان mRNA رن TGF-B1 در عضلات EDL و نعلى. 


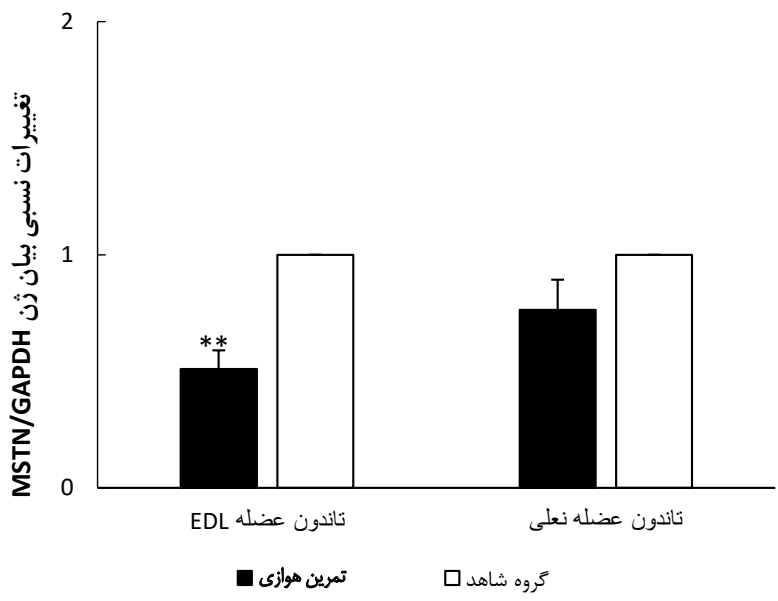

$$
\text { تصوير r. ميزان بيان mRNA رن ميوستاتين در عضلات EDL و نعلى }
$$$$
\text { PS } 1 . .1 \text {, «.* }
$$

غيرفعال سازى رنتيكى ميوستاتين در رتها ضمن افزايش حجم

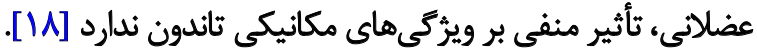

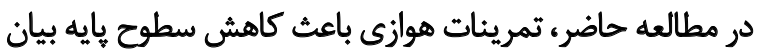

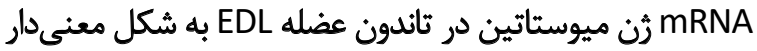

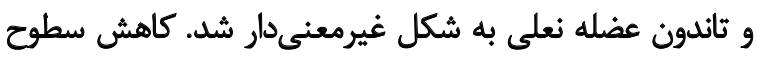

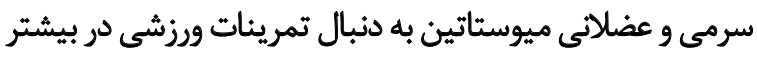

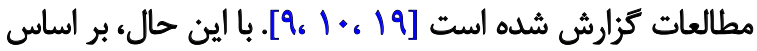

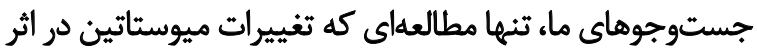

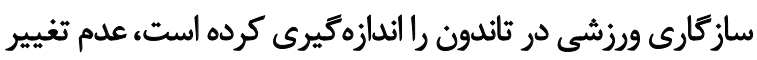

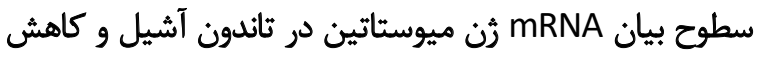

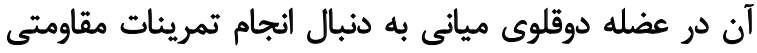

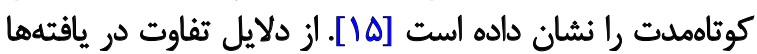

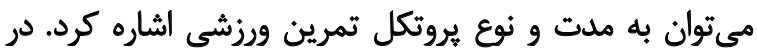

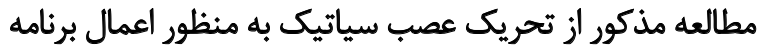

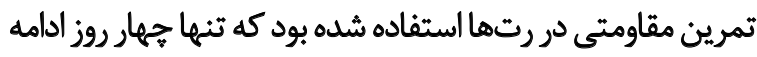
داشت، در حالى كه برنامه تمرين هوازى استفادهشده در مطالعه
آنتى اكسيدانى دارد و در ياسخ به التهاب عضله افزايش مي يابد. انجام تمرينات ورزشى در درازمدت قدرت آنتىاكسيداني عضله

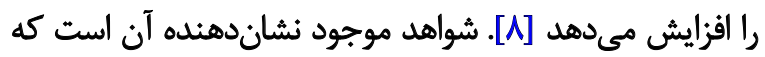

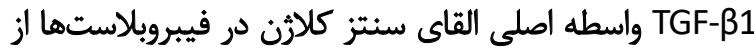

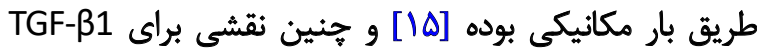

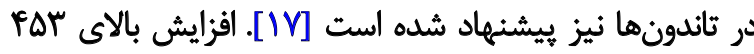

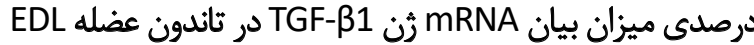

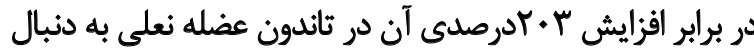

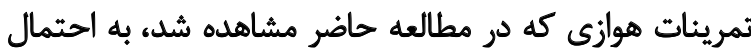

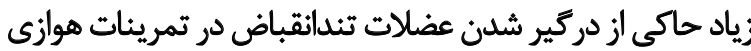
است كه در اعمال معمول روزانه كمتر مورد استفاده قرار مي تئنيرد. ميوستاتين علاوه بر اينكه اندازه، نوع و انقباضيذيرى عضله

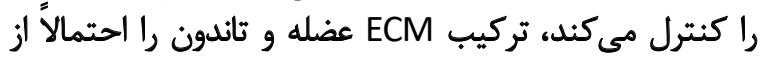

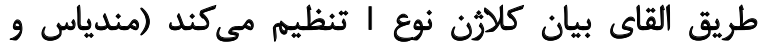

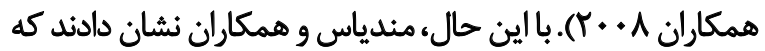

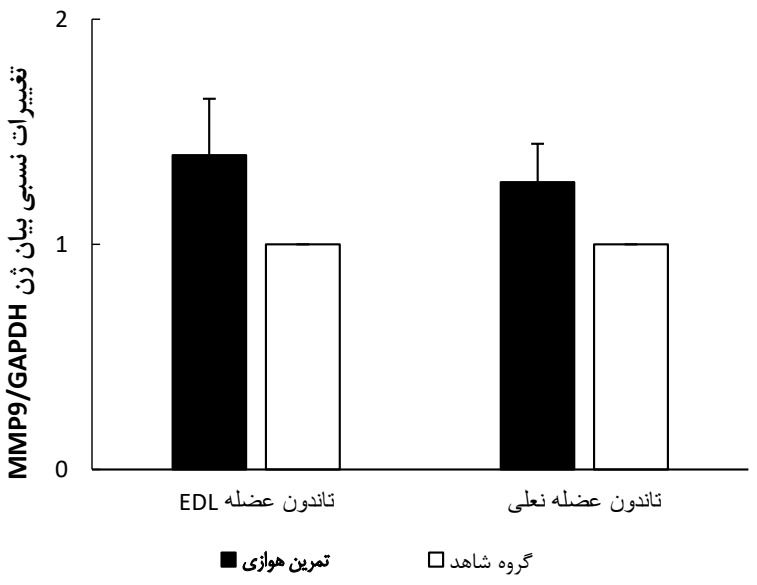

تصوير ّا. ميزان بيان MRNA رُ MPR9 در عضلات EDL و نعلى 


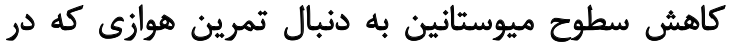

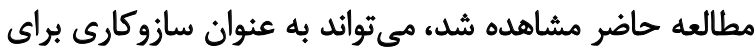

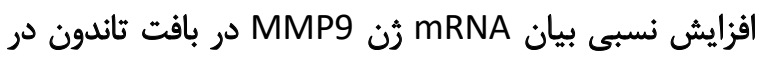

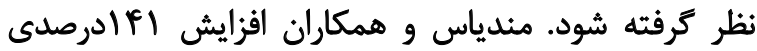

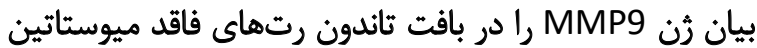

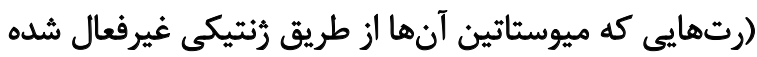

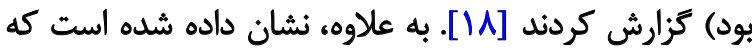

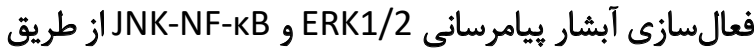

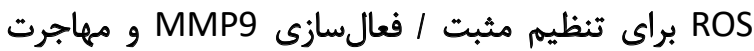

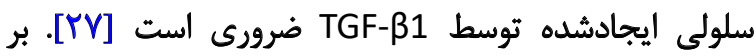

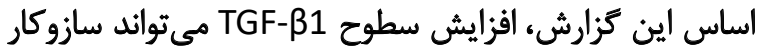

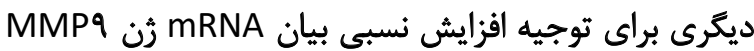
در بافت تاندون در اين مطالعه باشد.

$$
\text { نتيجلمَيرى }
$$

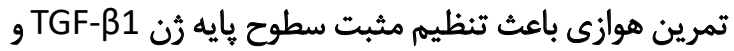

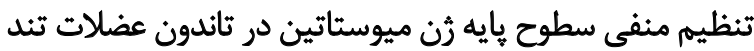

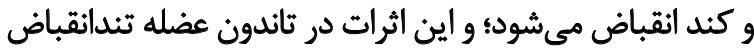

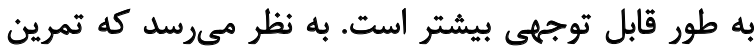

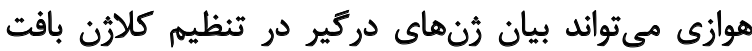

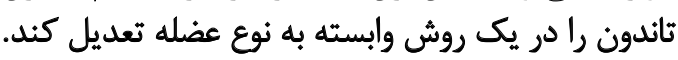

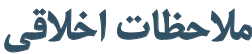

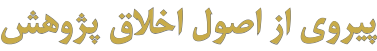

همه مراحل مربوط به كار با حيوانات با توجه به دستورالعمل

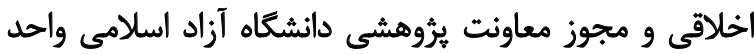
تهران مركزى با شماره IR.IAU.PS.REC.1398.296 انجام الخدام

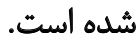

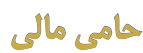

اين مقاله از رساله دكثرى نويسنده دوم، در كروه فيزيولوزي

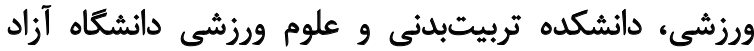
اسلامى واحد تهران مركزى استخراج شده است.

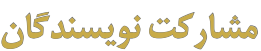

تعريف موضوع و بيان مسئله، نكارش متن و بازبينى: تمام نويسندكان؛ روش يثروهش و تحليل دادهها: قاسم محمدنثراد.

$$
\text { تقاوض مناقع }
$$

نويسندكان مقاله هيج كونه تعارضى در منافع اعلام نكردند.
حاضر دويدن روى تردميل به مدت شش هفته بود. به طور جالب، كزارش شده است كه نوع ورزش عامل اصلى ئى

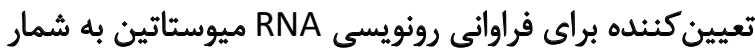

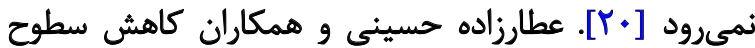

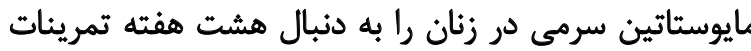

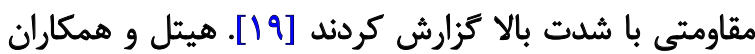

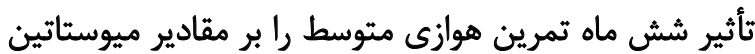

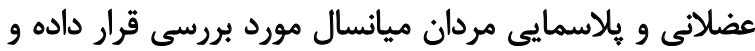

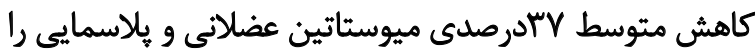

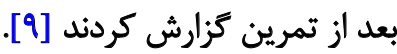

در تحقيقى ديكر ، ماتساكاس و همكاران نتايج متفاوتى رادر بادي

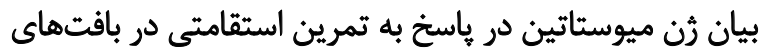

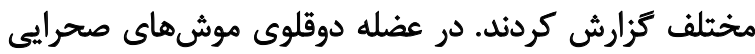

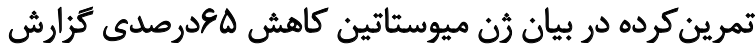

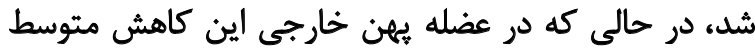

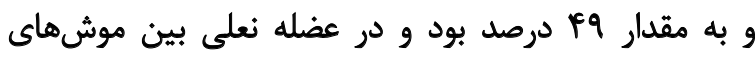

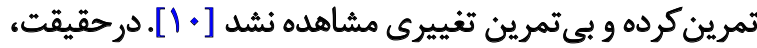

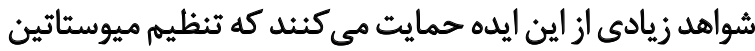

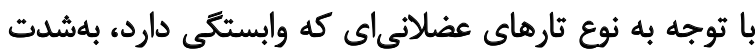

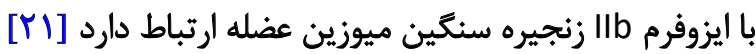

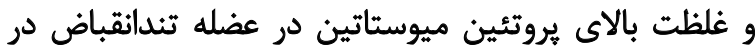

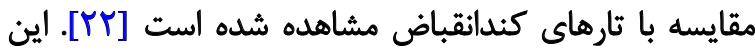

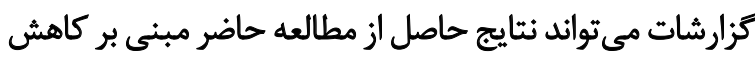

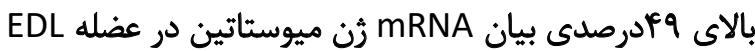

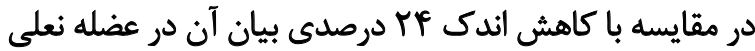
به دنبال تمرينات هوازى را توجيه كنيند.

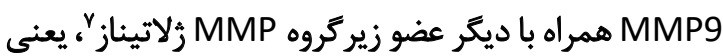

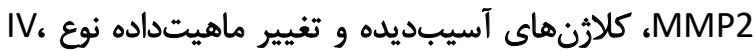

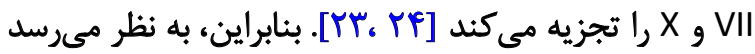

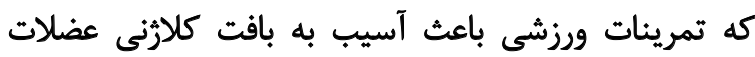

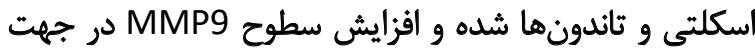

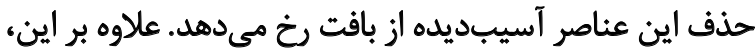

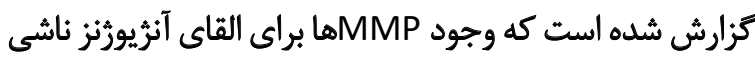

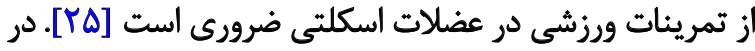

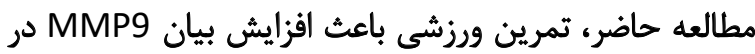

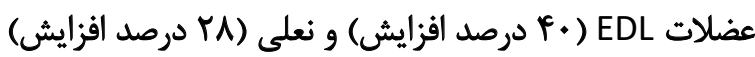

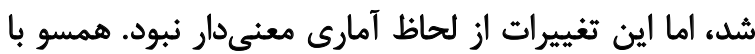

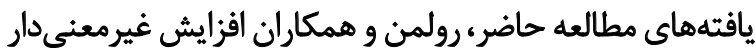

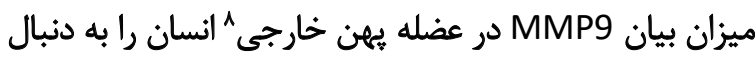

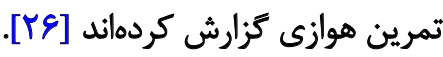




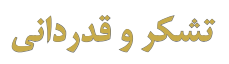

نويسندكان مقاله از جناب دكتر رضا قراخانلو، كروه

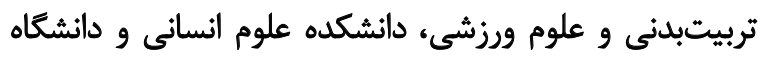

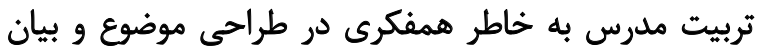
مسئله تقدير و تشكر مى كنيند. 
[1] Schiaffino S, Reggiani C. Molecular diversity of myofibrillar proteins: Gene regulation and functional significance. Physiol Rev. 1996; 76(2):371-423. [DOI:10.1152/physrev.1996.76.2.371] [PMID]

[2] Davis ME, Gumucio JP, Sugg KB, Bedi A, Mendias CL. MMP inhibition as a potential method to augment the healing of skeletal muscle and tendon extracellular matrix. J Appl Physiol. 2013; 115(6):884-91. [DOI:10.1152/ japplphysiol.00137.2013] [PMID] [PMCID]

[3] Kjær M, Langberg H, Heinemeier K, Bayer M, Hansen M, Holm L, et al. From mechanical loading to collagen synthesis, structural changes and function in human tendon. Scand J Med Sci sports. 2009;19(4):500-10. [DOI:10.1111/j.1600-0838.2009.00986.x] [PMID]

[4] McPherron AC, Lawler AM, Lee S-J. Regulation of skeletal muscle mass in mice by a new TGF-p superfamily member. Nature. 1997; 387(6628):83-90. [DOI:10.1038/387083a0] [PMID]

[5] Mendias CL, Bakhurin KI, Faulkner JA. Tendons of myostatin-deficient mice are small, brittle, and hypocellular. Proc Natl Acad Sci. 2008; 105(1):388-93. [DOI:10.1073/pnas.0707069105] [PMID] [PMCID]

[6] Mendias CL, Gumucio JP, Bakhurin KI, Lynch EB, Brooks SV. Physiological loading of tendons induces scleraxis expression in epitenon fibroblasts. J Orthop Res. 2012; 30(4):606-12. [DOI:10.1002/jor.21550] [PMID] [PMCID]

[7] Pryce BA, Watson SS, Murchison ND, Staverosky JA, Dünker N, Schweitzer R. Recruitment and maintenance of tendon progenitors by TGF $\beta$ signaling are essential for tendon formation. Dev. 2009; 136(8):1351-61. [DOI:10.1242/dev.027342] [PMID] [PMCID]

[8] Czarkowska-Paczek B, Zendzian-Piotrowska M, Bartlomiejczyk I, Przybylski J, Gorski J. The effect of acute and prolonged endurance exercise on transforming growth factor-beta1 generation in rat skeletal and heart muscle. J physiol pharmacol. 2009; 60(4):157-62. [PMID]

[9] Hittel DS, Axelson M, Sarna N, Shearer J, Huffman KM, Kraus WE. Myostatin decreases with aerobic exercise and associates with insulin resistance. Med Sci Sports Exerc. 2010; 42(11):2023. [DOI:10.1249/ MSS.0b013e3181e0b9a8] [PMID] [PMCID]

[10] Matsakas A, Friedel A, Hertrampf T, Diel P. Short-term endurance training results in a muscle-specific decrease of myostatin mRNA content in the rat. Acta Physiol Scand. 2005; 183(3):299-307. [DOI:10.1111/j.1365201X.2005.01406.x] [PMID]

[11] Gumucio JP, Sugg KB, Mendias CL. TGF- $\beta$ superfamily signaling in muscle and tendon adaptation to resistance exercise. Exerc sport Sci Rev. 2015; 43(2):93. [DOI:10.1249/JES.0000000000000041] [PMID] [PMCID]

[12] Koskinen SO, Heinemeier KM, Olesen JL, Langberg H, Kjaer M. Physical exercise can influence local levels of matrix metalloproteinases and their inhibitors in tendon-related connective tissue. J Appl Physiol. 2004; 96(3):861-4. [DOI:10.1152/japplphysiol.00489.2003] [PMID]

[13] Sadighi A AA, Azarbayjani MA, Barari AR. [Effect of aerobic exercise on some factors of cardiac apoptosis in male rats (Persian)]. Feyz. 2019; 23(5):495-502. http://feyz.kaums.ac.ir/article-1-3792-fa.html

[14] Pfaffl MW. A new mathematical model for relative quantification in real-time RT-PCR. Nucleic Acids Res. 2001; 29(9):e45-e. [DOI:10.1093/ nar/29.9.e45] [PMID] [PMCID]

[15] Heinemeier K, Olesen J, Haddad F, Langberg H, Kjær M, Baldwin K, et al. Expression of collagen and related growth factors in rat tendon and skeletal muscle in response to specific contraction types. J Physiol. 2007; 582(3):1303-16. [DOI:10.1113/jphysiol.2007.127639] [PMID] [PMCID]
[16] Czarkowska-Paczek B, Bartlomiejczyk I, Przybylski J. The serum levels of growth factors: PDGF, TGF-beta and VEGF are increased after strenuous physical exercise. J Physiol Pharmacol. 2006; 57:189-97. [PMID]

[17] Yang G, Crawford RC, Wang JH. Proliferation and collagen production of human patellar tendon fibroblasts in response to cyclic uniaxial stretching in serum-free conditions. J Biomech. 2004; 37(10):1543-50. [DOI:10.1016/j.jbiomech.2004.01.005] [PMID]

[18] Mendias CL, Lynch EB, Gumucio JP, Flood MD, Rittman DS, Van Pelt DW, et al. Changes in skeletal muscle and tendon structure and function following genetic inactivation of myostatin in rats. J Physiol. 2015; 593(8):2037-52. [DOI:10.1113/jphysiol.2014.287144] [PMID] [PMCID]

[19] Attarzadeh Hosseini SR, Moeinnia N, Motahari Rad M. The effect of two intensities resistance training on muscle growth regulatory myokines in sedentary young women. Obes Med. 2017; 5:25-8. [DOI:10.1016/j.obmed.2017.01.004]

[20] Matsakas A, Bozzo C, Cacciani N, Caliaro F, Reggiani C, Mascarello $F$, et al. Effect of swimming on myostatin expression in white and red gastrocnemius muscle and in cardiac muscle of rats. Exp Physiol. 2006; 91(6):983-94. [DOI:10.1113/expphysiol.2006.033571] [PMID]

[21] Carlson CJ, Booth FW, Gordon SE. Skeletal muscle myostatin mRNA expression is fiber-type specific and increases during hindlimb unloading. Am J Physiol-Regul Integr Comp Physiol. 1999; 277(2):R601-R6. [DOI:10.1152/ajpregu.1999.277.2.R601] [PMID]

[22] Wehling M, Cai B, Tidball JG. Modulation of myostatin expression during modified muscle use. FASEB J. 2000; 14(1):103-10. [DOI:10.1096/ fasebj.14.1.103] [PMID]

[23] Kim J, Lee J. Matrix metalloproteinase and tissue inhibitor of metalloproteinase responses to muscle damage after eccentric exercise. J Exerc Rehabil. 2016; 12(4):260-5. [DOI:10.12965/ jer.1632640.320] [PMID] [PMCID]

[24] Monaco S, Sparano V, Gioia M, Sbardella D, Di Pierro D, Marini S, et al. Enzymatic processing of collagen IV by MMP-2 (gelatinase A) affects neutrophil migration and it is modulated by extracatalytic domains. Protein Sci. 2006; 15(12):2805-15. [DOI:10.1110/ps.062430706] [PMID] [PMCID]

[25] Haas T, Milkiewicz M, Davis S, Zhou A, Egginton S, Brown M, et al. Matrix metalloproteinase activity is required for activity-induced angiogenesis in rat skeletal muscle. Am J Physiol-Regul Integr Comp Physiol. 2000; 279(4):H1540-H7. [DOI:10.1152/ajpheart.2000.279.4.H1540] [PMID]

[26] Rullman E, Norrbom J, Strömberg A, Wågsäter D, Rundqvist H, Haas T, et al. Endurance exercise activates matrix metalloproteinases in human skeletal muscle. J Appl Physiol. 2009; 106(3):804-12. [DOI:10.1152/japplphysiol.90872.2008] [PMID]

[27] Hsieh HL, Wang HH, Wu WB, Chu PJ, Yang CM. Transforming growth factor- $\beta 1$ induces matrix metalloproteinase- 9 and cell migration in astrocytes: roles of ROS-dependent ERK-and JNK-NF-KB pathways. J neuroinflammation. 2010; 7(1):88. [DOI:10.1186/1742-2094-7-88] [PMID] [PMCID] 
This Page Intentionally Left Blank 